\title{
Podocyte Damage in Acute Hypertensive Mice; Involvement of TRPC6
}

\author{
XIAOQIU WU AND BIN HUANG* \\ Department of Cardiovascular Medicine, The People's Hospital of Chongzuo, Chongzuo City, Guangxi Zhuang Autonomous \\ Region, China
}

Wu et al.: Acute Hypertensive Mice and TRPC6

\begin{abstract}
In order to study the effect of changes in TRPC6 protein pathway on podocyte injury in hypertensive mice, 20 WT mice were randomly divided into the control group and the experimental group. Acute hypertension model was established by ligating abdominal aorta for $15 \mathrm{~min}$ in the experimental group mice. After successful establishment of the mouse model, in vivo freezing technology is used in all mice to prepare tissue samples for the experiment. $\mathrm{H}$ and $\mathrm{E}$ staining, SABC-DAB immunohistochemical staining, Western blot and calcium ion detections were made on kidney tissue samples of mice. Urine samples of mice were collected to test urinary albumin. The results showed that compared to the control group, the glomerular tubules and Bowman capsules in the experimental group were swollen, the expression of TRPC 6 channels in podocytes increased, the concentration of calcium increased sharply and the concentration of albumin in urine also increased sharply. These results showed that hypertension could cause opening of TRPC6 channel to increase the calcium ion concentration leading to podocyte damage. The ability of damaged podocytes to deal with albumin is weakened and a large increase of albumin would cause further increase of $\mathrm{Ca}^{2+}$ concentration, that aggravated damage to podocytes this initiated a vicious circle. This study thus laid a foundation for exploring the mechanism of podocyte injury caused by hypertensions
\end{abstract}

Key words: Hypertension, mouse model, podocyte damage, TRPC6

Nowadays, the incidence of chronic kidney disease is increasing year by year all over the world, which brings more and more pressure to medical resources of various countries, which needs immediate attention of all parties ${ }^{[1,2]}$. In recent years, it has been recognized that podocyte injury is not only the initiating factor of chronic kidney disease, but also an important link leading to the progression of chronic kidney disease ${ }^{[3-5]}$. 
Therefore, in order to prevent the occurrence of the disease or delay the deterioration to reduce damage after the eventual inevitable illness, it is necessary to explore the relationship between podocyte injury and chronic kidney disease as much as possible, such as exploring the possible pathogenesis ${ }^{[6,7]}$. Podocytes play a very important role in glomerular filtration function. They form a filtration barrier and maintain the normal operation of renal function ${ }^{[8-10]}$. Podocytes are arranged along the lateral side of the glomerular basement membrane, hence are also called glomerular visceral epithelial cells ${ }^{[11]}$. Podocytes have a variety of functions and structures, but there is a transmembrane complex between adjacent podocytes, which is a molecular and charge barrier. Its function is to prevent the leakage of plasma proteins. At the same time, it acts as an important signal transduction platform in the podocytes ${ }^{[12,13]}$. TRP family are channel proteins, which are non-selective, receptor-gated cationic proteins. The expression of TRPC6 has been found in podocytes $^{[14]}$. Under certain conditions, especially when stimulated by external stimuli, TRPC6 in podocytes get activated leading to calcium ions entering or releasing from endoplasmic reticulum, and ultimately leading to podocyte damage ${ }^{[15]}$. TRPC6 channel plays an important role in regulating calcium signaling. The increase in intracellular $\mathrm{Ca}^{2+}$ regulated by TRPC6 is an important cause of podocyte injury ${ }^{[16]}$. Abnormal expression of TRPC6 is often found in podocyte injury. It was reported that focal segmental glomerulosclerosis (FSGS) often inherited by families could result from mutation of TRPC6. In acquired glomerulopathy, TRPC6 expression is abnormal, leading to $\mathrm{Ca}^{2+}$ influx, which then leads to activation of downstream signalling, rearrangement of podocyte skeleton and apoptosis of podocytes, leading to proteinuria ${ }^{[17-19]}$. Calcium influx can eventually lead to structural damage and dysfunction of podocytes. Based recent scientific advances, mitochondria have been found to play an increasingly important role in hypertension. In some cases, the changes of mitochondrial structure, especially the changes of mitochondrial DNA, are highly synchronous with the onset of hypertension ${ }^{[20]}$. Mitochondria are the main energy processing plants of human body, which provide more than $90 \%$ of the energy for the body. At the same time, they produce a large number of oxygen free radicals, induce apoptosis, and are related to a variety of cardiovascular diseases. It is suspected that TRPC6 regulates the increase of intracellular $\mathrm{Ca}^{2+}$ induced by acute hypertension, which leads to the overload of mitochondrial $\mathrm{Ca}^{2+}$ in podocytes, and then leads to the disintegration of mitochondria and apoptosis of podocytes ${ }^{[21]}$. In this study, in vivo freezing technology was used to better achieve the experimental objectives and obtain more accurate results. In vivo freezing technology is a rapid freezing technology for living organisms. The freezing rate of the technique is very fast and the tissue sample can be rapidly frozen to $-193^{\circ}$. In vivo freezing technology maintains the stable state and structural characteristics of TRPC6 in mouse glomerular podocytes. Real-time changes of TRPC6 protein in podocytes are obtained by using immunohistochemistry, immunofluorescence and Western blotting techniques. The changes of mitochondrial number and morphology in acute hypertension are observed under transmission electron microscopy so as to study the effects of hypertension on podocyte damage in mice.

Twenty healthy male wild (WT) mice weighing between 23-33 g, aged 6-8 w, were purchased from the Beijing Weitong Lihua Laboratory Animal Technology Co., Ltd., China. Feeding temperature was maintained at $20-26^{\circ}$. The mice were allowed free access to feed and water. Mice were randomly divided into 2 groups, 10 mice in each group. One group is the normal control group and the other group is the acute hypertension group (experimental group).

Establishment of an animal model of after anesthesia, the mice in the experimental group were placed on the anatomical plate in a supine position. The limbs were fully extended and fixed and the body was symmetrical. The skin was disinfected with alcohol, the skin and peritoneum were cut along the white line of the abdomen from the top of the pubic bone to the bottom of the sternum. The two ends of the incision were cut along the left and right directions to the ventral wall. The abdominal wall was reversed to the left and right sides, fully exposing the abdominal cavity. Intestines were gently pushed to the right with a small piece of absorbent paper to reveal the posterior abdominal wall. The adipose tissue behind the peritoneum is removed, to expose the abdominal aorta and main abdominal vein. The abdominal aorta was ligated for $15 \mathrm{~min}$ to establish the development of a hypertensive mouse model.

Mouse tissues were fixed using in vivo cryotherapy technology (IVCT). First, while the mouse abdominal aorta was ligated, propane-isopentane solution was prepared for instant freezing. Propane gas was slowly blown in to isopentane in a beaker and stirred while blowing to prevent the isopentane solution from freezing for $5 \mathrm{~min}$. After establishing the acute 
hypertension model (15 min after ligation of the abdominal aorta), the propane-isopentane mixture was immediately poured on to the exposed mouse kidneys. Mice were placed in a Styrofoam box containing liquid nitrogen. After the mice were fully frozen, kidney tissue samples were removed and stored. Firstly, 2\% paraformaldehyde acetone solution was prepared as follows, $20 \mathrm{~g}$ of dry paraformaldehyde was dissolved in $100 \mathrm{ml}$ of distilled water and mixed with acetone in the ratio 1:10 to obtain the replacement liquid, which was stored and frozen from light. Subsequently, the frozen mouse kidney was immersed in the replacement solution and the replacement solution was replaced every $12 \mathrm{~h}$. After $48 \mathrm{~h}$, the temperature was allowed gradually to return to room temperature and the next treatment was carried out. The sample was then treated with alcohol, acetone and xylene. The samples were the embedded in paraffin and sectioned. Part of the kidney tissue was taken out and placed in a $-20^{\circ}$ slicer chassis machine for rewarming. After $1 \mathrm{~h}$, the kidney tissue was sliced to obtain $8 \mu \mathrm{m}$ thick sections. The cut kidney sections were fixed in $4 \%$ formaldehyde solution. First, the paraformaldehyde-fixed frozen kidney tissue sections were taken out and washed with tap water for 1-2 min. The tissue sections were blotted dry and stained with hematoxylin for 50-60 s. The sections were washed with tap water for 10-15 s to remove hematoxylin dye solution. Tissue sections were then differentiated with $1 \%$ saline ethanol for 1-2 min and washed with tap water for 1-2 min. Next, these were immersed in saturated $\mathrm{Li}_{2} \mathrm{CO}_{3}$ solution for 7-10 $\mathrm{s}$ and immediately rinsed in running water for $30 \mathrm{~s}$. Finally, the tissue sections were stained with eosin for $1 \mathrm{~min}$. After staining gradient ethanol was used to dehydrate with $70,80,95 \%$ and absolute ethanol for 1-3 min. Sections were soaked in xylene I for $1 \mathrm{~min}$, soaked in xylene II for $1 \mathrm{~min}$ and sealed with a neutral gum for imaging. The kidney paraffin sections were placed on a baking machine for 30 min to melt the wax on the sections. Tissue sections were washed twice with PBS for $5 \mathrm{~min}$ each time. Distilled water was used to prepare fresh 3\% $\mathrm{H}_{2} \mathrm{O}_{2}$, which was incubated for $10 \mathrm{~min}$ at room temperature in deionized water. Distilled water was used for washing 3 times for antigen retrieval. PBS was used to wash for 5 min. Normal goat serum blocking solution was added dropwise. After $20 \mathrm{~min}$ at room temperature, excess liquid is removed. $60 \mu 1 \mathrm{I}$ antibody was added dropwise to each section and incubated overnight at $4^{\circ}$. After incubation, rewarmed to $37^{\circ}$ for 45 min, washed thrice with PBS for 2 min each time. Biotinylated secondary antibody was added dropwise, incubated at $37^{\circ}$ for $30 \mathrm{~min}$, washed 3 times with PBS for 2 min each time. SABC reagent was added dropwise, incubated at $37^{\circ}$ for $30 \mathrm{~min}$ and washed 4 times with PBS for 5 min each time. Color was developed using a $\mathrm{DAB}$ chromogenic kit and the reaction time was controlled under a microscope. After the developer was added dropwise, it was stained at room temperature for $4 \mathrm{~min}$. The tissue sections were washed with distilled water, counterstained with hematoxylin for about $30 \mathrm{~s}$ and washed with distilled water. The final slice was processed through alcohol, xylene and hydrochloric acid alcohol for differentiation, xylene dehydration and clarification and subjected to neutral gelatin seal microscopy. The remaining frozen kidney tissue samples were taken out on ice and $200 \mathrm{mg}$ of each sample was placed in a EP tube and minced on ice. Then, $1 \mathrm{ml}$ of PBS at $4^{\circ}$ was added, shaken gently on a shaker, placed in a cryogenic centrifuge, and centrifuged at $3000 \mathrm{rpm}$ for $4 \mathrm{~min}$ at $4^{\circ}$. The supernatant was discarded, the pellet was washed with PBS twice, centrifuged and the supernatant was discarded. In the precipitation, $0.4 \mathrm{ml}$ RIPA pyrolysis solution (PMSF 10 $\mu 1+$ protease inhibitor $5 \mu \mathrm{l}$ ) was added and ultrasonicated on ice followed by centrifugation for $15 \mathrm{~min}$ at 15000 rpm for $4^{\circ}$ in a refrigerated centrifuge. The supernatant obtained was the extract of cytoplasmic protein. The supernatant was transferred to a tube and sealed with a sealing film, labelled and stored at $-80^{\circ}$. Protein concentration in the supernatant was determined using BCA protein quantitation kit (Pierce). The protein content of the supernatant was mixed with the same amount of sample buffer, boiled and centrifuged at 10 $000 \mathrm{rpm}$. After $10 \mathrm{~min}$, the upper supernatant was taken and stored. Samples containing equal amounts of protein were sampled on to SDS- polyacrylamide gel (SDS-PAGE) for vertical electrophoresis (separation gel $8 \%$, concentrated glue $5 \%$ ) and marker was used as a control. The electrophoresis is terminated when the protein reached the bottom of the gel. The total protein of the tissue in gel swimming lane was transferred to PVDF membrane by semi dry transfer electrophoresis. After successful transmembrane transfer, the membrane was cut into 2 parts according to the Marker's size and the internal reference protein and the target protein band were separated and labelled. Then, the membrane was sealed overnight at $4^{\circ}$ in the sealing solution containing $5 \%$ skimmed milk powder and washed with TBST for $5 \mathrm{~s}$. Then, the internal reference protein membrane and the target protein membrane were incubated at room temperature for $2 \mathrm{~h}$ in $1 \% \mathrm{BSA}$ diluted internal reference solution and 1\% BSA diluted 
GIRK4 antibody solution, respectively. TBST was used to wash the membranes for 5 times, 10 min each time. The reference protein membrane the target protein membrane were placed in 1\% BSA diluted GADPH antibody solution and 1\% BSA diluted GIRK4 antibody solution, respectively and incubated at room temperature for $2 \mathrm{~h}$. The membranes were washed with TBST for 5 times, 10 min each time. ECL kit darkroom shadow, $\mathrm{X}$-ray film exposure and film development to show GIRK4 and glyceraldehyde-3 acid dehydrogenase (GADPH) protein bands. In the gel imager, Quantity One software was used to quantitatively analyze the gray level of GIRK4 and GADPH protein bands, and the relative expression intensity of GIRK4 protein was expressed by the ratio of the target protein of GIRK4 protein to the gray value of GADPH band. Nephron sections were processed before routine immunostaining, or after immunostaining, subsequent Hoechst staining was performed. PBS was used to wash twice, $3 \mathrm{~min}$ each time and the liquid is aspirated. When washing, both table concentrator and manual shaking for several times was used. It was done in a 6 well plate. Hoechst 33258 dye solution $0.5 \mathrm{ml}$ was added to stain for $5 \mathrm{~min}$. PBS or $0.9 \% \mathrm{NaCl}$ was used to wash twice for $3 \mathrm{~min}$ each time. The slices were placed on the slide, a drop of antiquenching sealing liquid was added, covered with a clean cover glass to avoid bubbles as far as possible. The blue nuclei were detected under fluorescence microscopy. The excitation wavelength was $350 \mathrm{~nm}$ and the emission wavelength was $460 \mathrm{~nm}$. The absorption and emission spectra of Hoechst 33258 were the left peak and the right peak were the emission spectra. Intracellular calcium concentration was expressed by relative fluorescence intensity (real-time fluorescence intensity/basic fluorescence intensity). Calcium imaging was performed at room temperature within $1 \mathrm{~h}$ after incubation with Fluo3-AM. The concentration of urinary albumin in the collected urine samples of mice was detected by enzyme-linked immunosorbent assay (ELISA). The calculation of urinary albumin excretion rate was based on the concentration and content of urinary albumin measured by ELISA kit. The unit of urinary albumin value was expressed in $\mu \mathrm{g}$. Statistical analysis is performed by SPSS24.0 software. Quantitative data conforms to normal distribution and were expressed as mean \pm standard deviation (SD). Student'st test is used for comparison. Rates are compared using Chi-square $\left(\mathrm{X}^{2}\right)$ test. $\mathrm{P}<0.05$ is defined as difference with statistical significance. In the control group, the capillary surface of renal capsule was smooth, red blood cells were fixed in it, blood vessels were full and fissured Bowman sac was visible. In the experimental group, although red blood cells were still fixed in the capillary, they were not as full as those in the control group and swelling Bowman sac and renal tubule were visible at the same time, as shown in fig. 1. The distribution of TRPC6 in glomerular podocytes is shown in fig. $2 \mathrm{~A}$ and $\mathrm{B}$. It was observed that TRPC6 protein was expressed along the capillary loops of the glomeruli of mice under normal blood flow conditions, which was generally continuous granular distribution. However, in acute hypertension, TRPC6 began to appear in the cytoplasm and translocated to the perinuclear and intranuclear areas. The expression of TRPC6 in the capillary loops was significantly reduced and distributed intermittently as shown in fig. $2 \mathrm{C}(\mathrm{P}<0.05)$. Western blot results showed that the expression of TRPC6 protein in the podocytes of the control group was significantly higher than that of the experimental group, as shown in fig. 2D and $\mathrm{E}$ $(\mathrm{P}<0.05)$. Calcium ions was detected in the cytoplasm of podocytes of both the groups of mice. It was found that in acute hypertensive stimulation can increase the concentration of free $\mathrm{Ca}^{2+}$ in the cytoplasm of podocytes. The fluorescence images of calcium ions of the control and the experimental groups were shown in fig. 3A and $\mathrm{B}$, respectively. On comparing the relative fluorescence intensity of calcium ions, it was found that there was a significant difference in the concentration of calcium ions between the two groups $(\mathrm{P}<0.05)$. Albumin is the main protein component of proteinuria. Albumin is often used as the representative to study the damage of proteinuria to the innate cells of kidney. Previous studies on the effects of albumin on the kidney have focused on the damage to the proximal tubular epithelium. After the detection of urinary albumin in both groups of mice, it was found that the concentration of urinary albumin in the experimental group increased sharply, which indicated that acute hypertension could

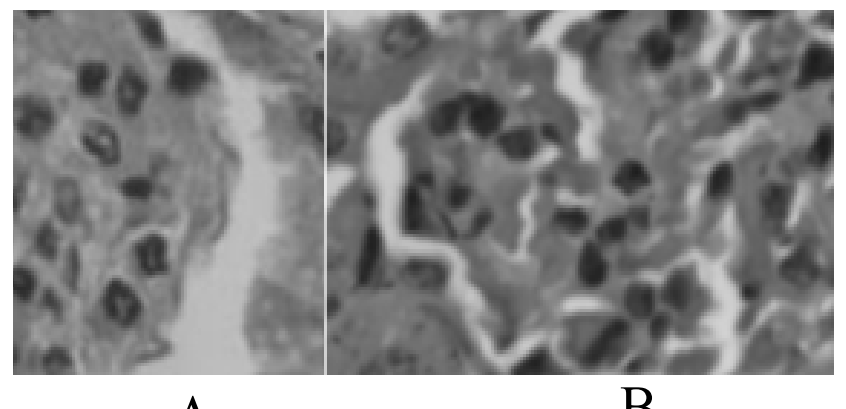

A B

Fig. 1: $H$ and $E$ stained kidney sections

$H$ and $E$ staining of kidney sections mice under different hemodynamic conditions, A. under normal blood flow condition and $B$. under acute hypertension 


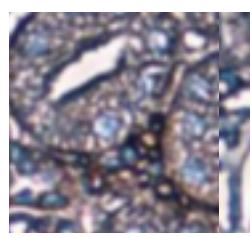

A

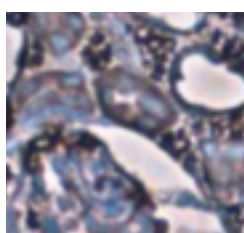

B

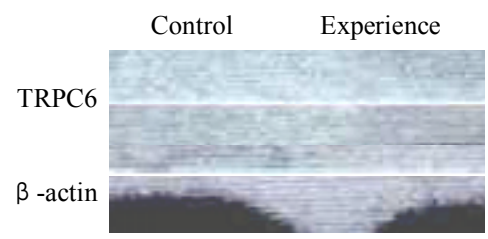

$\mathrm{D}$

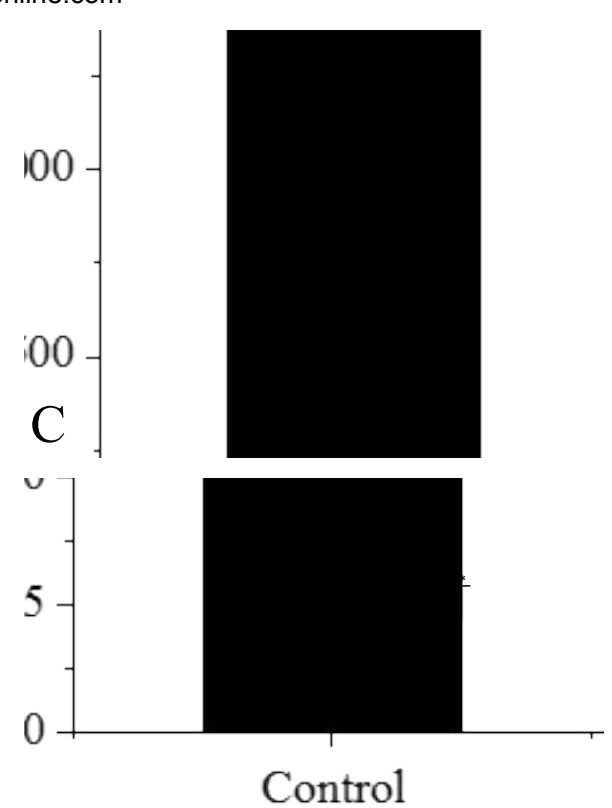

$\mathrm{E}$

Fig. 2: Expression of TRPC6 protein

Expression of TRPC6 protein as shown by immunohistochemical staining in A. control group, B. experimental group. C. Comparison of IOD values of TRPC6 in vascular loops between the two groups, D. Electrophoresis map of TRPC6 protein expression in the two groups and E. Comparison of TRPC6 protein expression level in podocytes of the two groups

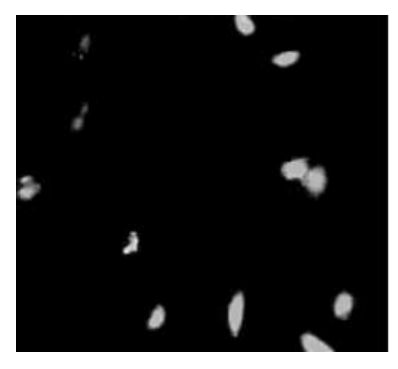

A

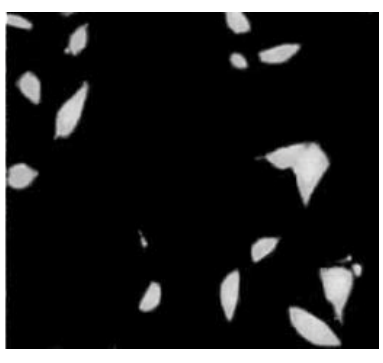

B

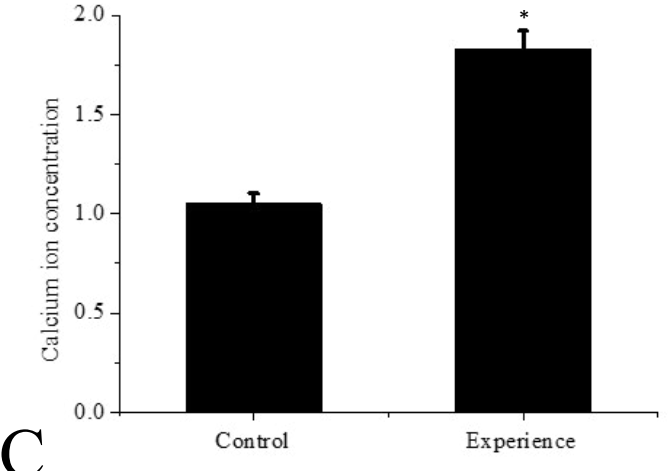

Fig. 3: Calcium ion concentration through fluorescence imaging Calcium ion concentration through fluorescence imaging in A. control group B. experimental group and C. comparison of calcium ion concentration between the two groups

significantly increase the concentration of urinary albumin in mice $(\mathrm{P}<0.05)$. Albumin could aggregate in the subpodal space of podocytes. If it exceeds the ability of podocytes to deal with albumin, it would cause damage, and the ability of damaged podocytes to deal with albumin would further decrease, thus causing a vicious circle. A large amount of albumin could stimulate the $\mathrm{Ca}^{2+}$ concentration in podocyte cytoplasm to increase. This might be one of the mechanisms by which albumin directly damages podocytes. In abnormal hemodynamics, especially acute hypertension, the risk of kidney damage increases. Traditional kidney removal and fixation methods cannot capture the transient changes of renal fine structure and TRPC6 expression caused by these sudden changes in hemodynamics. Therefore, in this experiment, in vivo freezing technology was used to better achieve the experimental purpose and obtain more accurate results. Conventional treatment techniques often fail to maintain the stability of tissue and cell microstructures, while in vivo freezing techniques can overcome the shortcomings of conventional techniques as much as possible. More importantly, on the premise of using cryopreservation technology in vivo, further studies such as $\mathrm{H}$ and $\mathrm{E}$ staining and immunohistochemistry can be performed in the following experiments, which could help to observe the changes of renal cell microstructure and provide more accurate conclusions for future research. 
www.ijpsonline.com
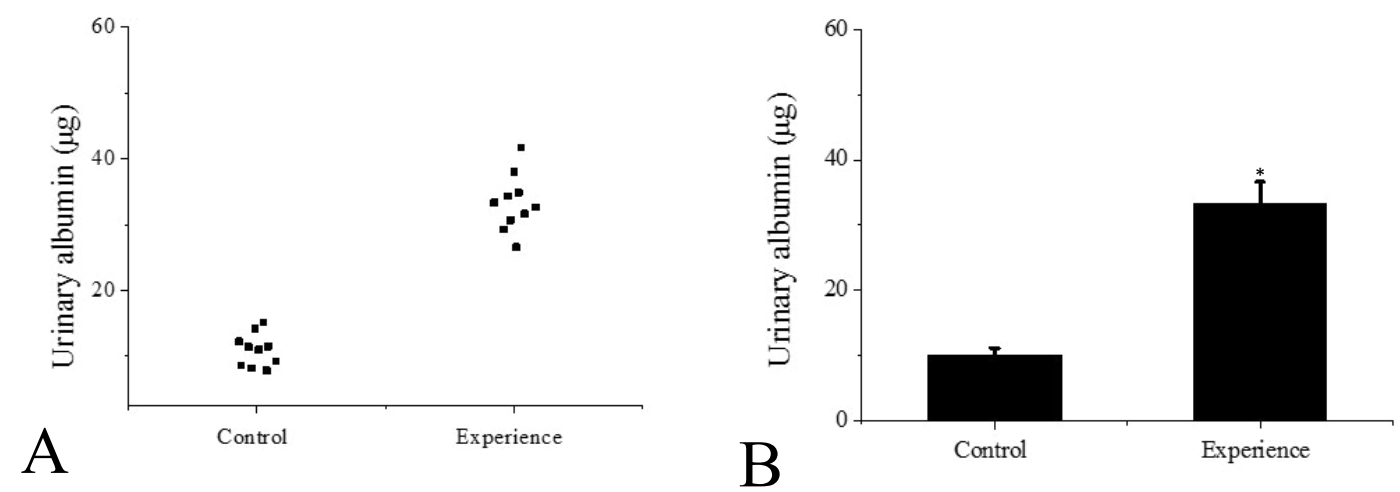

Fig. 4: Urinary albumin levels in both groups of mice Urinary albumin levels of mice as A. a scatter plot and B. Comparison of urinary albumin concentration between both the groups

In the study, TRPC6 expression increased as well as $\mathrm{Ca}^{2+}$ influx. Thus, TRPC6 channel opening is strengthened in hypertensive state and TRPC6 channel affects hypertension by regulating calcium concentration. Hypertension enhanced the opening of TRPC6 channels, which led to a sharp increase in $\mathrm{Ca}^{2+}$ concentration, resulting in podocyte damage. The ability of damaged podocytes to deal with albumin reduced. A large increase in albumin also causes an increase in $\mathrm{Ca}^{2+}$ concentration, which further damages podocytes.

\section{REFERENCES}

1. Abraham VC, Miller LN, Pratt SD. Implementation of a human podocyte injury model of chronic kidney disease for profiling of renoprotective compounds. Eur J Pharmacol 2017;815:21932.

2. Hayek SS, Koh KH, Grams ME. A tripartite complex of suPAR, APOL1 risk variants and $\alpha v \beta 3$ integrin on podocytes mediates chronic kidney disease. Nat Med 2017;23(8):945.

3. Rota C, Morigi M, Cerullo D. Therapeutic potential of stromal cells of non-renal or renal origin in experimental chronic kidney disease. Stem Cell Res Ther 2018;9(1):220.

4. Wu W, Yang JJ, Wan YG. Pathogenesis and treatment of insulin resistance in chronic kidney disease and interventional effects of Chinese herbal medicine. China J Chinese Materia Medica 2017;42(1):49-55.

5. Bruni R, Possenti P, Bordignon C. Ultrasmall polymeric nanocarriers for drug delivery to podocytes in kidney glomerulus. J Control Release 2017;255:94-107.

6. Yu J, Feng Q, Wong SH. Metagenomic analysis of faecal microbiome as a tool towards targeted non-invasive biomarkers for colorectal cancer. Gut 2017;66(1):70-8.

7. Hahm E, Wei C, Fernandez I, Bone marrow-derived immature myeloid cells are a main source of circulating suPAR contributing to proteinuric kidney disease. Nat Med 2017;23(1):100-06.

8. Xu MX, Wang M, Yang WW. Gold-quercetin nanoparticles prevent metabolic endotoxemia-induced kidney injury by regulating TLR4/NF- $\kappa \mathrm{B}$ signaling and Nrf2 pathway in high fat diet fed mice. Int J Nanomed 2017;12:327-45.

9. Michishita R, Matsuda T, Kawakami S. Hypertension and hyperglycemia and the combination thereof enhances the incidence of chronic kidney disease (CKD) in middle-aged and older males. Clin Exp Hypertens 2017;39(37):1.

10. Wong C, Gange SJ, Buchacz K. First occurrence of diabetes, chronic kidney disease, and hypertension among North American HIV-infected adults, 2000-2013. Clin Infect Dis 2017;64(4):ciw804.

11. Kushiro T, Kario K, Saito I. Increased cardiovascular risk of treated white coat and masked hypertension in patients with diabetes and chronic kidney disease: the HONEST Study. Hypertens Res Official J Japanese Soc Hypertens 2017;40(1):87-95.

12. Weir MR, Mayo MR, Garza D. Effectiveness of patiromer in the treatment of hyperkalemia in chronic kidney disease patients with hypertension on diuretics. J Hypertens 2017;35 (Suppl 1):S57-S63.

13. Kanbay M, Onal EM, Afsar B. The crosstalk of gut microbiota and chronic kidney disease: role of inflammation, proteinuria, hypertension, and diabetes mellitus. Int Urol Nephrol 2018; $1-14$.

14. Asghari G, Yuzbashian E, Mirmiran P. The association between Dietary Approaches to Stop Hypertension and incidence of chronic kidney disease in adults: the Tehran Lipid and Glucose Study. Nephrol Dial Transplant 2017;32(suppl2):ii224-ii230.

15. Nijenhuis T, Sloan AJ, Hoenderop JG. Angiotensin II contributes to podocyte injury by increasing TRPC6 expression via an NFAT-mediated positive feedback signalling pathway. Am J Pathol 2011;179(4):1719-32.

16. Kim EY, Roshanravan H, Dryer SE. Changes in podocyte TRPC channels evoked by plasma and sera from patients with recurrent FSGS and by putative glomerular permeability factors. BBA Mol Basis Disease 2017;1863(9):2342-54.

17. Ilatovskaya DV, Blass G, Palygin O. A NOX4/TRPC6 Pathway in Podocyte Calcium Regulation and Renal Damage in Diabetic Kidney Disease. J Am Soc Nephrol 2018;29(7):ASN.2018030280.

18. Zhang L, Ji T, Wang Q. Calcium-Sensing Receptor Stimulation in Cultured Glomerular Podocytes Induces TRPC6-Dependent Calcium Entry and RhoA Activation. Cell Physiol Biochem 2017;43(5):1777-89.

19. Guan Y, Nakano D, Zhang Y. A protease-activated receptor-1 antagonist protects against podocyte injury in a mouse model of nephropathy. J Pharmacol Sci 2017;135(2).

20. Liu B, He X, Li S. Deletion of diacylglycerol-responsive TRPC genes attenuates diabetic nephropathy by inhibiting 
www.ijpsonline.com

activation of the TGF $\beta 1$ signaling pathway. Am J Transl Res 2017;9(12):5619-30.

21. Urban N, Neuser S, Hentschel A. Pharmacological inhibition of FSGS-related TRPC6 gain of function mutants by semisynthetic larixol-derived compounds. $\mathrm{Br} \mathrm{J}$ Pharmacol 2017;11(6):5960.

22. Cao S, Liu YG. Research advances in the association between transient receptor potential cation channel 6 and kidney disease. Chinese J Contemporary Pediatrics 2018;20(1):72-6..
This is an open access article distributed under the terms of the Creative Commons Attribution-NonCommercial-ShareAlike 3.0 License, which allows others to remix, tweak, and build upon the work non-commercially, as long as the author is credited and the new creations are licensed under the identical terms

This article was originally published in Special issue on "Trends in therapeutic Management of Various Conditions" Indian J Pharm Sci 2020:82(3) 